\title{
Incidence, electrophysiological characteristics, and long-term follow-up of perimitral atrial flutter in patients with previously confirmed mitral isthmus block
}

\author{
Panagiotis Ioannidis $^{1}$, Evangelia Christoforatou ${ }^{1}$, Theodoros Zografos ${ }^{1}$, Panagiotis \\ Charalambopoulos $^{1}$, Konstantinos Kouvelas ${ }^{1}$, Theodora Kappou ${ }^{1}$, Andreas Tsoumeleas ${ }^{1}$, \\ Sotirios Floros ${ }^{1}$, Dimitrios Tagoulis ${ }^{1}$, Ioannis Ntarladimas ${ }^{1}$, Maria Kalantzi ${ }^{1}$, Ioannis \\ Tagoulis $^{2}$, Elias Eleftheriades ${ }^{1}$, Dimitrios Avzotis ${ }^{1}$, Antonis Manolis ${ }^{3}$, and Charalambos \\ Vassilopoulos $^{1}$ \\ ${ }^{1}$ Athens Bioclinic \\ ${ }^{2}$ Red Cross Hospital \\ ${ }^{3}$ Athens University School of Medicine
}

October 20, 2020

\begin{abstract}
Introduction: After mitral isthmus (MI) catheter ablation, perimitral atrial flutter (PMF) circuits can be maintained due to the preservation of residual myocardial connections, even if conventional pacing criteria for complete MI block are apparently met (MI pseudo-block). We aimed to study the incidence, the electrophysiological characteristics, and the long-term outcome of these patients. Methods and Results: Seventy-two consecutive patients (mean age $62.4 \pm 10.2,62.5 \%$ male) underwent MI ablation, either as part of an atrial fibrillation (AF) ablation strategy $(n=35)$, or to treat clinical reentrant atrial tachycardia (AT) $(n=32)$, or to treat AT that occurred during ablation for AF $(n=5)$. In all patients the electrophysiological characteristics of PMF circuits were studied by high-density mapping. MI block was successfully achieved in 69/72 patients (95.6\%). Five patients developed PMF after confirming MI block. In these patients, high-density mapping during the PMF showed a breakthrough in MI with extremely low impulse conduction velocity $(\mathrm{CV})$. In contrast, in usual PMF circuits that occurred after AF ablation, the lower CV of the reentrant circuit was of significantly higher value $(0.07 \pm 0.02 \mathrm{~m} / \mathrm{s}$ vs $0.25 \pm 0.07 \mathrm{~m} / \mathrm{s}$, respectively; $\mathrm{P}<0.001)$. Patients presented with clinical AT had better prognosis in maintaining sinus rhythm after MI ablation compared with patients presented with AF. Conclusion: PMF with MI pseudo-block may be present after MI ablation and has specific electrophysiological features characterized by remarkably slow CV in the MI. Thus, even after MI block is achieved, a more detailed mapping in the boundaries of the ablation line or reinduction attempts may be needed to exclude residual conduction.
\end{abstract}

\section{INTRODUCTION}

The mitral isthmus (MI) is the anatomical area between the ostium of the left inferior pulmonary vein (PV) and the posterolateral part of the mitral valve annulus. Mitral isthmus ablation is an established strategy in the treatment of perimitral atrial flutter (PMF) [1, 2] as well as an adjunct to pulmonary vein isolation (PVI) in the treatment of non-paroxysmal atrial fibrillation (AF) $[3,4]$.

The usefulness of the MI ablation and generally of linear lesions is questionable as an initial strategy in persistent AF ablation [5,6]. However, it is one of the possible options in cases where PVI alone is not considered sufficient, such as in redo cases with well-maintained PVI [7], long-lasting persistent AF [8,9], or diseased myocardium with low-voltage electrical activity [10]. On the other hand, MI ablation is an effective 
therapy for PMF, however, these reentrant tachycardias can also be treated by ablating critical isthmuses with slow conduction zones [11-13].

If a linear lesion is attempted in the MI, the creation of complete and bidirectional block is very important. Failure to achieve bidirectional block can be proarrhythmic and therefore if it cannot be achieved it should not even be attempted [14-16]. The anatomical complexity of the MI hinders the creation of transmural lesions [17]. This can lead to the persistence of conduction gaps that allow the maintenance of PMF despite the apparent evidence of complete block. This is a condition often referred to as MI pseudo-block [18-21].

In this prospective study, we investigated clinical and electrophysiological characteristics of patients who had undergone MI ablation, focusing in particular on the group of patients who continued to have PMF, while MI block had been previously demonstrated by evidentiary pacing maneuvers. At the same time, we tried to peruse standard PMF circuits by measuring conduction velocities, in order to understand the specific electrophysiological properties of these reentrant tachycardias and to improve our knowledge in selecting the most appropriate PMF ablation strategy.

\section{METHODS}

\section{Patient population}

The study population comprised consecutive patients who underwent MI catheter ablation, from January 2018 to January 2020, either as part of a specific AF ablation strategy, or to treat clinical reentrant AT, or to treat AT that occurred during ablation for AF. All patients underwent baseline evaluation with clinical history, physical examination, electrocardiogram, and a transthoracic echocardiogram. The definitions and terminology regarding $\mathrm{AF}$ in this article are in agreement with the latest HRS/EHRA/ECAS/APHRS/SOLAECE Expert Consensus Statement on Catheter and Surgical Ablation of AF [22]. All patients provided written informed consent. The study protocol was approved by the hospital's institutional review board. The study complied with the Declaration of Helsinki.

\subsection{Electrophysiological study}

All antiarrhythmic medications except for amiodarone were discontinued at least 5 half-lives before ablation. All patients were on oral anticoagulant therapy which was not interrupted for the procedure. For patients taking vitamin $\mathrm{K}$ antagonists an international normalized ratio between 2.0 and 3.0 was a prerequisite for the preoperative period as well as for the day of the procedure. For patients taking direct oral anticoagulants with twice-daily dosing, the morning dose was also given in the majority of the patients, however, in a small minority a single dose was held on the day of the procedure. Transesophageal echocardiography was performed in all patients just before the start of the procedure to exclude left atrial (LA) thrombi. The ablation procedure was performed under deep sedation with propofol, fentanyl and midazolam.

Twelve-lead ECG and intracardiac electrograms were continuously monitored and stored on a computerbased digital amplifier/ recorder system (EP Workmate Recording System; Abbott, St Paul, MN, USA). Intracardiac electrograms were filtered from 30 to $500 \mathrm{~Hz}$. The EnSite Precision three-dimensional system (Abbott, St Paul, MN, USA) was used for mapping of the left and the right atrium if appropriate.

Barium ingestion, before the general anesthesia, was used for real-time fluoroscopic imaging of the esophagus. A $100 \mathrm{IU} / \mathrm{kg}$ dose of heparin was administered immediately after the insertion of the femoral sheaths. Subsequent boluses of heparin were administered every 1 hour targeting an activated clotting time $>300 \mathrm{~s}$.

The following diagnostic catheters were introduced via the femoral veins: (1) a quadripolar catheter was positioned in the His bundle area; (2) a deflectable decapolar 6F catheter, electrode spacing 2-5-2 mm (Inquiry L, Abbott) was positioned in the coronary sinus (CS), with the distal electrode reaching the 4 o'clock position along the mitral annulus in the $30^{\circ}$ left anterior oblique (LAO) radiographic projection.

A transseptal puncture was carried out with the use of an intracardiac echocardiography (ICE) catheter (ViewFlex Xtra, Abbott) connected with a compatible ultrasound console (Philips CX50). A 20-pole, variable radius circumferential mapping catheter (Reflexion Spiral, Abbott) was introduced with the help of a long 
transseptal sheath (Preface, Biosense Webster, or LAMP 45 , Abbott) to guide PVI, geometry creation, and high-density activation and voltage mapping.

A contact force ablation catheter (TactiCath Sensor Enabled, Abbott) was inserted into the left atrium (LA) through a steerable transseptal sheath (Agilis $71 \mathrm{~cm}$, Abbott). An anatomical three-dimensional LA model was systematically created by first inserting the ablation catheter in all PVs and the LA appendage (LAA), and then, by precisely delineating the body of the LA with the circumferential mapping catheter.

For the electrophysiological evaluation of Ats, local activation time (LAT) and propagation maps, as well as conventional entrainment mapping were used to clarify the AT mechanism. A focal AT was defined when the activation map was indicative of centrifugal activation from a distinct source, while a macro-reentrant tachycardia was diagnosed with 3D propagation maps when a discrete impulse rotation was evident corresponding to the entire cycle length (CL) of the tachycardia. Conventional entrainment mapping contributed to the diagnosis or was omitted if the propagation map was definitively diagnostic.

\section{Catheter ablation}

At the initial AF ablation procedure, a wide antrum PVI was attempted. After placing the 20-polar circumferential PV mapping catheter in each PV, a point-by-point circumferential lesion encompassing the ipsilateral PVs was created aiming at complete electrical PV disconnection. RF energy was applied with an upper power of $30 \mathrm{~W}$, a temperature limit of $40 \mathrm{C}^{\circ}$ and saline irrigation flow $17 \mathrm{ml} / \mathrm{min}$ during RF delivery. A force of 10-30g and a force-time integral (FTI) $>400 \mathrm{gs}$ was targeted in every ablation lesion. If the ablation catheter was in close proximity to the esophagus, which was fluoroscopically visible with barium, the power was reduced to $25 \mathrm{~W}$. In cases of non-paroxysmal AF, after PVI, additional ablation of complex fractionated atrial electrograms or linear lesions were attempted at the discretion of the operators.

At the redo procedures, the PV antra were meticulously re-mapped for conduction recurrence and if observed, additional lesions were administered, using the above ablation settings, to achieve PVI. Previously administered linear lesions were also checked for bidirectional block, and in the case of residual conduction, further lesions were delivered. If the recurrent clinical arrhythmia was persistent AF, beyond the re-isolation of the PVs, box lesion in the LA posterior wall, MI ablation and possibly cavo-tricuspid isthmus (CTI) ablation were performed. If the clinical arrhythmia was AT, the mechanism of the tachycardia was targeted in an individualized manner. For focal tachycardias, the area of origin was ablated, while for macro-reentrant tachycardias, complete linear lesions were performed in anatomical isthmuses (MI, LA roof, CTI).

\section{Mitral isthmus ablation}

The MI ablation was preferably performed in sinus rhythm (SR). Special emphasis was given on the accuracy of LA geometry in the MI area, and if there were obvious deficiencies, points were re-collected to correct the 3D model. The MI line started at the 4 o'clock position of the mitral annulus (LAO view) and ended at the lower portion of the circular isolation line of the left PVs. With the ablation catheter supported by the steerable sheath a contact force of $15-35 \mathrm{~g}$ was applied in every ablation point. If the achieved pressure was less than $15 \mathrm{~g}$, the ablation lesion was not attempted to avoid edema which could potentially hinder the formation of a transmural lesion. The radiofrequency (RF) application started from the ventricular aspect of the mitral annulus at the point where the atrial potential was apparent. The RF energy was delivered with an FTI target of 400-1000 gs at each point, until the elimination of local electrogram or its reduction by $90 \%$. After the completion of MI linear ablation, point by point mapping was performed along the MI line and reinforcement ablation was given to any site with residual high-frequency potentials or electrograms of an amplitude $>0.05 \mathrm{mV}$. After elimination of the atrial potentials from the endocardial site, and if complete block had not been achieved, the ablation catheter was inserted through the CS reaching from the epicardium the level of the endocardial MI line. At this point and if discrete atrial potential was recorded, RF energy was delivered for maximum $15 \mathrm{~s}$, with contact force of less than $30 \mathrm{~g}$, power of $25 \mathrm{~W}$, a temperature limit of $40 \mathrm{C}^{\circ}$ and saline irrigation flow $17-30 \mathrm{ml} / \mathrm{min}$. The energy delivery at the epicardial site was continued until complete block attainment or elimination of the atrial potentials. 
The complete and bidirectional MI block was verified by pacing maneuvers. For the implementation and interpretation of the pacing maneuvers, special care was taken to ensure that the pacing and recording catheters were as close as possible to the MI line. By pacing at CL of $500-600 \mathrm{~ms}$ from the LAA or a point superiorly and close to the line, we sought complete and distinct reversal of the activation sequence in the CS. Additionally, differential pacing from the distal and an adjacent CS bipole would confirm conduction block in the counterclockwise direction (Figure 1 ). When the MI line was made during PMF, the termination of the tachycardia was further confirmatory of the PMF diagnosis, but the complete MI block had to be proven in SR by the pacing maneuvers described above. In all patients, the endocardial MI line length (longitudinal distance from mitral annulus to left inferior PV), and MI line width (maximum transverse distance from the anterior to posterior border of the ablated area) were measured, on the geometry created by the 3-D system. The endocardial and epicardial RF ablation time were also measured.

\subsection{Conduction velocity (CV) measurement}

After the construction of the LAT map and the confirmation of the PMF diagnosis, a careful observation of the propagation map was carried out in order to identify areas with apparent delay throughout the whole wavefront course in a complete tachycardia circle. In these areas, a manual point correction was carried out at each received point, by locating the first deflection of the local electrogram. The timing scale was set either by spreading the color palette over the entire CL of the tachycardia or by defining color changing steps of $20 \mathrm{~ms}$. In the derived map the shortest transverse distance in the direction of the wavefront propagation of an isochronal step was measured. For CV measurement, this distance was divided by the time interval of isochronal step and expressed in $\mathrm{m} / \mathrm{s}$. From the analyzed areas, the sites with the lower CV were pointed out. In the inspection of the propagation map, channels of conduction with a width [?]1 $\mathrm{cm}$ were sought, and the impulse CV was also measured in these channels. Eligible for this analysis were maps containing more than 2,000 points with sufficient dispersion throughout the circuit course.

\section{Follow-up}

After ablation, antiarrhythmic drugs could be administered at the discretion of the electrophysiologists for a period of 2-3 months. Anticoagulants were continued for the first 2-3 months and stopped when the CHA2DS2VASC score was $<1$. All patients underwent follow-up at 1, 3, 6, and 12 months following the ablation procedure and yearly thereafter. During the entire post-procedural period, the patients were also followed by their referring physicians. Subsequent information was obtained by phone and/or e-mail. Follow-up visits included clinical examination, an ECG recording, and 24-h Holter monitoring. Tachycardia recurrence was defined as any atrial tachyarrhythmia (AT or AF) episode lasting [?] $30 \mathrm{~s}$ documented in an ECG or Holter recording or during interrogation of an implantable cardiac rhythm device. A 3-month time interval after MI ablation was defined as blanking period.

\section{Statistical analysis}

Continuous variables are summarized as mean \pm standard deviation (SD) or as median (interquartile range) according to data normality and were compared using Student's t test or Mann-Whitney rank-sum test, as appropriate. Categorical data are summarized as frequencies and percentages and were compared using Pearson Chi-squared test and Fisher's exact test. Between-group differences were assessed using one-way ANOVA or the Kruskal-Wallis test, according to data normality. Rates for freedom from arrhythmia recurrence were determined using Kaplan-Meier analysis and were compared by the log-rank test across groups. Data analyses were performed using IBM SPSS Statistics version 25 (Armonk, NY, USA). A p value $<0.05$ was considered statistically significant.

\section{RESULTS}

\subsection{Patient characteristics and interventional outcome}

Seventy-two consecutive patients underwent MI catheter ablation either as part of a specific AF ablation strategy $(n=35)$, or to treat clinical reentrant AT $(n=32)$, or to treat AT occurred during ablation for AF $(\mathrm{n}=5)$. Patient baseline characteristics are shown in Table $\mathbf{1}$. According to the predefined pacing maneuvers 
(Figure 1 ), the MI block was successfully achieved in 69 out of $72(95.8 \%)$ patients (in 68 after the first procedure and in 1 patient after the second procedure). Of the four patients in whom complete MI block was unsuccessful during the first procedure, one had hypertrophic cardiomyopathy (MI block was achieved during the second ablation procedure), one suffered from cardiac amyloidosis (diagnosed later), while in the other two, the access to CS was not achieved due to a CRT-D left ventricular lead, and a particular anatomical configuration, respectively. Of the three patients whose MI block was not finally achieved, only one had PMF treated with an anterior linear lesion at the same procedure, and since then the patient remains in SR. The mean MI ablation time of was $10.73 \pm 6.16$ min which was consisted of $10.12 \pm 5.87$ min for endocardial ablation and $1 \pm 0.54$ min for epicardial ablation through the CS.

\subsection{Incidence of PFM with MI pseudo-block}

Despite the confirmation of MI block with the aforementioned pacing maneuvers, 5 patients presented with PMF. In 3 of these patients, PMF was detected during the MI ablation procedure, while 2 patients had recurrence at 3 and 6 months after the MI ablation procedure, and the diagnosis of PMF was made during the redo procedure, while pacing maneuvers were false-positive for MI block before any ablation attempt (Figure 2 ). In all 5 patients the detailed mapping showed a MI isthmus breakthrough with slow conduction along a narrow channel (Figure 3, Videos 1-4 ). The endocardial ablation at this area restored the completeness of the MI line in all these patients. The complete and bidirectional block of the MI was reevaluated by pacing maneuvers and careful endocardial and epicardial mapping in the boundaries of the ablation line. In addition, after programmed atrial stimulation, PMF was no longer inducible.

\subsection{PMF electrophysiological characteristics}

During the procedures, 32 PMF (25 clockwise and 7 counterclockwise) were detected in 28 patients (24 with AT and 4 with AF as the clinical arrhythmia). In 20 of the 32 PMF circuits (16 clockwise and 4 counterclockwise) it was possible to construct a high-density map and measure the CVs as described earlier (Figure 4, Videos 5,6 ). The mean CL of PMFs with pseudo-block (all clockwise) did not differ significantly from the mean CL of usual PMFs ( $254 \pm 10 \mathrm{~ms}$ vs $248 \pm 13 \mathrm{~ms}$, respectively; $\mathrm{P}=0.340$ ). However, the lower $\mathrm{CV}$ in PMFs with pseudo-block was of significantly lower value in comparison with the lower CV in other thoroughly mapped PMF circuits $(0.07 \pm 0.02 \mathrm{~m} / \mathrm{s}$ vs $0.25 \pm 0.07 \mathrm{~m} / \mathrm{s}$, respectively; $\mathrm{P}<0.001)$. The same was true for patients with PMF who had undergone additional LA ablation beyond PVI (Figure 5 ). Conduction channels with width [?]1 $\mathrm{cm}$ were found in 3 patients with PMF without MI pseudo-block. In these channels the mean CV was $0.34+-0.08 \mathrm{~m} / \mathrm{s}$ (range: $0.25-0.4 \mathrm{~m} / \mathrm{s}$ ) (Figure 6, Video 7 ). In patients with PMF and MI pseudo-block the comparison of the MI length as well as the MI width of the ablation area showed no difference compared to patients with successful MI ablation. However, the width of the MI ablation area was significantly longer in patients with unsuccessful MI ablation (Table 2 ).

\subsection{Arrhythmia recurrences and details of the redo procedures}

After the index procedure which included MI ablation, a redo procedure was required in 9 patients. The $\mathrm{AF} / \mathrm{AT}$ free survival rate, in a mean follow-up period of $22.2 \pm 8.1$ months, was $66.7 \%$ (48/72 patients) and $77.8 \%$ (56/72 patients) after the index MI ablation procedure and the supplementary redo procedure, respectively. Particularly, in patients with AT, the event-free survival rate was $71.9 \%$ (23/32 patients) and $87.5 \%$ (28/32 patients) after the index and the redo procedure, respectively, while patients with AF had $62.5 \%$ (25/40 patients) and 70\% (28/40 patients) event-free survival rate after the index and the redo procedure, respectively. Patients with AT appeared to have a significantly better prognosis in maintaining SR after MI ablation than patients with AF (log-rank, $\mathrm{P}=0.0466$ ) (Figure 7 ). However, 87.5\% of patients with AT had previously undergone LA ablation compared to $22.5 \%$ of patients with AF. Of the 4 patients who had unsuccessful MI ablation at the initial procedure, one who relapsed with PMF has undergone re-ablation with successful completion of MI and since then he is in SR, while of the other three, two had AF recurrence and underwent rate control therapy and one remained in SR. Among patients with PMF and MI pseudo-block, only one recurred with a brief episode of paroxysmal AF 18 months after the index MI ablation procedure.

After the successful MI ablation, 9 patients underwent a redo procedure due to AT/AF recurrence. In $8 / 9$ 
(88.9\%) the pacing proof of MI block was maintained. In one of the 9 patients the MI conduction had overtly recurred, and in 2 patients MI pseudo-block was confirmed, as the pacing criteria of MI block were fulfilled in parallel with the occurrence of PMF. The PMF was successfully treated by ablating the gap in MI.

Thirty-seven patients who underwent MI ablation had previous AF ablation (33 one procedure, and 4 two procedures, 1.1 procedures / patient). During the MI ablation procedure, PV reconnection was found in 27/37 (73\%) patients with at least 1 PV reconnected and in 66/148 (44.6\%) of the re-evaluated PVs.

\subsection{Complications}

In our series we had only one major complication. A 47-year-old male patient, who was ablated for longstanding persistent AF, 15 days after the procedure, being on rivaroxaban, presented with cardiac tamponade and moderate left pleural effusion. The patient had undergone his first AF ablation in which PVI, box lesion and successful MI ablation (without CS epicardial access) was performed. The patient was promptly treated with pericardiocentesis, and after a short hospital stay, he fully recovered. To date he is free of any atrial arrhythmia recurrence for a follow-up period of 29 months.

\section{DISCUSSION}

\subsection{Main findings}

1. After MI ablation, despite the evidence of complete and bidirectional MI block demonstrated by conventional pacing maneuvers, it is possible for PMF to occur at a rate of $6.9 \%$, as shown in our series.

2. The acute success of MI ablation is high, as shown in our study, where $77.8 \%$ of patients remained free of arrhythmia at a mean follow-up period of $22.2 \pm 8.1$ months, with a significantly better prognosis in the group of patients whose clinical arrhythmia was AT compared with those presented with AF.

3. The lower CV in PMFs with MI pseudo-block is significantly lower than the lower CV in usual PMF circuits and also in PMF circuits of patients who have previously undergone additional ablation in the anterior LA.

\subsection{Interventional outcome}

In our series, we had a satisfactory acute success rate ( $\sim 96 \%)$ in achieving complete MI block. Probably, our systematic approach with the steerable sheath support, the continuous maintenance of sufficient catheter contact force $(>15 \mathrm{~g})$ and the accuracy of the 3D imaging - there were no patient movements due to general anesthesia - contributed to this high percentage [23]. However, as shown by other studies [24], there will always be a considerable group of patients in whom we cannot achieve complete MI block with the available technological means. Thus, in our study, if we add the percentage of interventional failure $(4.2 \%)$ and the percentage of patients presenting with PMF and MI pseudo-block (6.9\%) we reach an $11.1 \%$. These patients have a higher likelihood to develop PMF even if this arrhythmia is not pre-existing, a fact that we need to take into consideration when we intend to perform the MI line [14-16]. Presumably, reinduction attempts should be performed only to exclude PMF induction after each successful MI ablation. On the contrary, the reinduction of AF, despite some relatively contradictory data [25], does not seem to be a predictor in maintaining SR [26]. In our study, we also found that MI ablation is a time-consuming procedure with the mean pure ablation time being almost 11 minutes, something that we must always take into account for the preparation of the procedural plan.

\subsection{Technical aspects of MI ablation}

In the process of MI ablation, the most prominent demonstration of successful completion of the linear lesion is the clear reversal of the activation sequence in the CS during pacing from the LAA. For the MI block confirmation we used pacing criteria indicative of bidirectional change in the activation sequence of the peri-mitral region and not simply the increase in MI conduction time or the recording of double potentials which are more uncertain criteria [27]. It seems that, in the same manner as in CTI ablation, the activation sequence shifts abruptly [28]. In about half of the cases, epicardial access was required in our study, the sequence in CS was reversed with minimal energy delivery within the CS. The extent of the lesions delivered 
epicardially through the CS does not anatomically extend to the entire epicardial aspect, obviously because there is no free access to a much wider area, but only to the muscular bundles surrounding the CS. This may create a selective block towards the CS multipolar catheter but not to the entire MI. Presumably, some cardiac bundles may remain intact, and as we have learned, these may be located in the myocardial coverage of the vein of Marshall, the complex anatomical region of the LA ridge, the lateral aspect of the LAA base, or the area between the LAA and the mitral annulus [18-21]. Apparently, the impulse is passing through the remaining MI connections with a very long delay. Due to this fact we have an apparent change in activation sequence as the impulse from pacing beside the line is delayed in its passage through the MI lesions going much faster in the opposite direction (Figure 8 ).

In the study by Barragan et al. [18] it was shown that the breakthroughs are in the majority (8/11) epicardial. In our study all the PMFs with MI pseudo-block were ablated from the endocardial site. This can be explained in three ways: a) the conduction gap is located in the endocardial site, b) more endocardial ablation is needed to create a transmural lesion, or c) that we finally ablate the endocardial breakthroughs of epicardial connections [19], although the critical lesions, in our 5 cases, were located in juxtaposition with the MI line. In the study of Fujisawa et al. [20], the pseudo-block was observed in $33.3 \%$ of the cases. In this study, the MI ablation was attempted at a higher level in contrast to our series where the initial choice was the 4 o'clock site of the mitral annulus (LAO view), possibly lower than the position of the ligament of Marshall and posteriorly of the lateral border of the LAA. If the MI line cannot be performed, alternatively other linear lesions were attempted to interrupt MI conduction or PMF circuits $[29,30]$, something that we attempted in our study in case of failure to achieve complete MI block. Probably, one way to avoid residual MI connections, beyond the re-induction challenge mentioned above, is the detailed mapping in the boundary of the line and the identification of near field potentials representing conduction gaps.

\subsection{Conduction velocities and implications for PMF ablation}

In our study we showed that PMF in patients with MI pseudo-block is an arrhythmia with special electrophysiological features. The evaluation of the activation maps revealed a very slow conduction through a narrow excitable channel in the area of the previous lesions. On the contrary, measuring the CV with high-density mapping in a large number of PMF that occur after AF ablation, we found that the CV does not show large differences throughout the course of the circuit. This also applies to patients with PMF who have had previous ablation in LA beyond PVI. In addition, the presence of conduction channels was found in a very small percentage of PMF circuits. Therefore, it appears that in the majority of PMFs after AF ablation there are areas where the CV is slower, but usually there is no area with marked conduction delay. This reasonably raises the question of what the most secure strategy for PMF ablation could be to ensure no recurrence. If we target the area of the lowest CV or the narrowest corridor, we can temporarily stop the circuit but there may still be pathways throughout the extent of the perimitral area that may allow its re-initiation. Nevertheless, it is doubtful whether the area around a channel in a propagation map is truly unexcitable or could become excitable in a different waveform direction. Thus, it seems reasonable that the most secure way to prevent PMF is to interrupt the circuit in the MI. Perhaps a useful example is the ablation of CTI-dependent flutter, where the arrhythmia can be terminated even with the onset of ablation, if a critical area is affected, but there is no doubt that the circuit can relapse unless complete and bidirectional CTI block is eventually achieved.

In several recent series with high-density mapping, targeting the functional rather than the anatomical isthmus has been the main ablation strategy [11-13]. It should be considered that many of these studies $[12,13]$ have been performed with the Rhythmia system, which actually creates high density maps, but probably does not facilitate the linear ablation with complete and transmural lesions, as this system did not have catheters with contact force technology. According to our previous observation in a longitudinal patient cohort with ATs after AF ablation, targeting the macro-reentrant circuits of the LA with ablation of the anatomical isthmuses has better results in maintaining SR [31]. However, we must recognize that all these views are based on empirical observations. Besides, the question of whether we should ablate the anatomical or the functional isthmus is difficult to answer even by a randomized study, as each case has specific and 
unique features and so it is difficult to implement a preplanned ablation strategy.

\subsection{Long term outcome}

Our study showed that patients with AT had a better prognosis in maintaining SR than those with AF. Evidently, it should not be overlooked that patients with AT had a much higher rate of previous LA ablation than patients with AF. Apparently, during the AT ablation, apart from the MI, additional ablation was carried out to treat other ATs or to re-isolate the PVs. Probably, when AT becomes a clinical arrhythmia after AF ablation, a less complex substrate is created, which if mapped in detail, can be ablated with great success and favorable long-term results [32].

In the redo cases we found the pacing-proven maintenance of MI line at a rate of $86 \%$. On the other hand, the maintenance rate of PVI per patient with at least one PV reconnected and per PV was $27 \%$ and $55 \%$, respectively, after 1.1 previous AF ablation procedures. Our findings are consistent with the study by Mujiovic et al. [33] in which the invasive re-evaluation, 3 months after the index procedure, showed numerically higher maintenance rate in linear lesions than in PVI. We do not know why this happens. It is possible that the greater persistence on a limited surface and the definite procedural endpoint in MI ablation lead to more transmural lesions.

\subsection{Limitations}

In our study we found a significant proportion of patients with PMF after conventional proof of complete block. However, we cannot ignore the fact that there may be cases with pseudo-block without subsequent PMF recurrence, as the re-induction challenge was performed only after AT ablation and not systematically in the context of AF ablation.

Regarding patients who developed PMF after confirmation of MI block in the same procedure, the recurrence of MI conduction in an "on-off" manner probably cannot be excluded, although the pacing confirmation was always performed very close to PMF occurrence. On the other hand, patients who developed PMF in another procedure and were found from the beginning to meet the pacing criteria for MI block should confidently be considered to have MI pseudo-block.

Another limitation is that the CV measurement is governed by subjective selection of the acquired points. By taking different points for this measurement or even by shifting the timing of the isochronal scale it can lead to significant differences in the CV value. However, all these measurements were made in highdensity activation maps, a fact that mitigates the subjectivity in CV evaluation. In addition, the significant difference found between PMFs with MI pseudo-block and usual PMFs that occur after AF ablation probably circumvents the above limitation.

\section{CONCLUSION}

MI ablation is a process that, if performed diligently, achieves a high rate of acute success. In a small percentage of patients, probably due to the anatomical complexity of MI, incomplete ablation lines could permit the occurrence of PMF circuits despite the verisimilar proof of MI block. These tachycardias are reentrant circuits characterized by a large deceleration of CV in the area of previous lesions in the MI. In this particular feature they differ from ordinary PMF circuits, in which the CV does not show huge differences in the whole extent of their course. This fact is probably advocating for ablation directed at anatomical rather than functional isthmuses.

Moreover, in our study we found that patients who underwent MI ablation and had AT, usually after previous AF ablation, had a much better prognosis than those who underwent MI ablation presenting with AF. Probably, after a sufficient substrate modification the less complex electrophysiological environment can only maintain ATs, which if mapped in detail and treated in a tailored manner, are very likely not to recur.

\section{REFERENCES}


1. Matsuo S, Wright M, Knecht S, et al. Peri-mitral atrial flutter in patients with atrial fibrillation ablation. Heart Rhythm. 2010;7(1):2-8. doi:10.1016/j.hrthm.2009.09.067

2. Dukkipati S, Holmvang G, Ruskin J, Mansour M. Ablation of mitral isthmus-dependent atrial flutter using integration of real-time electroanatomic mapping with three-dimensional computed tomography. Heart Rhythm. 2006;3(1):124. doi:10.1016/j.hrthm.2005.06.024

3. Okamatsu H, Okumura K. Strategy and Outcome of Catheter Ablation for Persistent Atrial Fibrillation - Impact of Progress in the Mapping and Ablation Technologies. Circ J. 2017;82(1):2-9. doi:10.1253/circj.CJ-17-1205

4. Lo LW, Lin YJ, Chang SL, Hu YF, Chung FP, Chen SA. Beyond Pulmonary Vein Isolation: the Role of Additional Sites in Catheter Ablation of Atrial Fibrillation. Curr Cardiol Rep. 2017;19(9):86. Published 2017 Aug 9. doi:10.1007/s11886-017-0884-4

5. Verma A, Jiang CY, Betts TR, et al. Approaches to catheter ablation for persistent atrial fibrillation. N Engl J Med. 2015;372(19):1812-1822. doi:10.1056/NEJMoa1408288

6. Sau A, Al-Aidarous S, Howard J, et al. Optimum lesion set and predictors of outcome in persistent atrial fibrillation ablation: a meta-regression analysis. Europace. 2019;21(8):1176-1184. doi:10.1093/europace/euz108

7. Gianni C, Anannab A, Della Rocca DG, et al. Recurrent Atrial Fibrillation with Isolated Pulmonary Veins: What to Do. Card Electrophysiol Clin. 2020;12(2):209-217. doi:10.1016/j.ccep.2020.02.001

8. Haïssaguerre M, Sanders P, Hocini M, et al. Catheter ablation of long-lasting persistent atrial fibrillation: critical structures for termination. J Cardiovasc Electrophysiol. 2005;16(11):1125-1137. doi:10.1111/j.1540-8167.2005.00307.x

9. Mody BP, Raza A, Jacobson J, et al. Ablation of long-standing persistent atrial fibrillation. Ann Transl Med. 2017;5(15):305. doi:10.21037/atm.2017.05.21

10. Sim I, Bishop M, O'Neill M, Williams SE. Left atrial voltage mapping: defining and targeting the atrial fibrillation substrate. J Interv Card Electrophysiol. 2019;56(3):213-227. doi:10.1007/s10840-019-005378

11. Sundaram S, Choe W, Ryan Jordan J, et al. Catheter ablation of atypical atrial flutter: a novel 3D anatomic mapping approach to quickly localize and terminate atypical atrial flutter. J Interv Card Electrophysiol. 2017;49(3):307-318. doi:10.1007/s10840-017-0269-z

12. Winkle RA, Moskovitz R, Mead RH, et al. Ablation of atypical atrial flutters using ultra high densityactivation sequence mapping. J Interv Card Electrophysiol. 2017;48(2):177-184. doi:10.1007/s10840016-0207-5

13. Laţcu DG, Bun SS, Viera F, et al. Selection of Critical Isthmus in Scar-Related Atrial Tachycardia Using a New Automated Ultrahigh Resolution Mapping System. Circ Arrhythm Electrophysiol. 2017;10(1):e004510. doi:10.1161/CIRCEP.116.004510

14. Sawhney N, Anousheh R, Chen W, Feld GK. Circumferential pulmonary vein ablation with additional linear ablation results in an increased incidence of left atrial flutter compared with segmental pulmonary vein isolation as an initial approach to ablation of paroxysmal atrial fibrillation. Circ Arrhythm Electrophysiol. 2010;3(3):243-248. doi:10.1161/CIRCEP.109.924878

15. Anousheh R, Sawhney NS, Panutich M, Tate C, Chen WC, Feld GK. Effect of mitral isthmus block on development of atrial tachycardia following ablation for atrial fibrillation. Pacing Clin Electrophysiol. 2010;33(4):460-468. doi:10.1111/j.1540-8159.2009.02625.x

16. Sawhney N, Anand K, Robertson CE, Wurdeman T, Anousheh R, Feld GK. Recovery of mitral isthmus conduction leads to the development of macro-reentrant tachycardia after left atrial linear ablation for atrial fibrillation. Circ Arrhythm Electrophysiol. 2011;4(6):832-837. doi:10.1161/CIRCEP.111.964817

17. Wittkampf $\mathrm{FH}$, van Oosterhout MF, Loh $\mathrm{P}$, et al. Where to draw the mitral isthmus line in catheter ablation of atrial fibrillation: histological analysis. Eur Heart J. 2005;26(7):689-695. doi:10.1093/eurheartj/ehi095

18. Barkagan M, Shapira-Daniels A, Leshem E, Shen C, Anter E. Pseudoblock of the Posterior Mitral Line With Epicardial Bridging Connections Is a Frequent Cause of Complex Perimitral Tachycardias. Circ Arrhythm Electrophysiol. 2019;12(1):e006933. doi:10.1161/CIRCEP.118.006933 
19. Chen S, Zhou G, Lu X, et al. The importance of identifying conduction breakthrough sites across the mitral isthmus by elaborate mapping for mitral isthmus linear ablation. Europace. 2019;21(6):950-960. doi:10.1093/europace/euy327

20. Fujisawa T, Kimura T, Nakajima K, et al. Importance of the vein of Marshall involvement in mitral isthmus ablation. Pacing Clin Electrophysiol. 2019;42(6):617-624. doi:10.1111/pace.13640

21. Takatsuki S, Fukumoto K, Igawa O, et al. Ridge-related reentry: a variant of perimitral atrial tachycardia. J Cardiovasc Electrophysiol. 2013;24(7):781-787. doi:10.1111/jce.12120

22. Calkins H, Hindricks G, Cappato R, et al. 2017 HRS/EHRA/ECAS/APHRS/SOLAECE expert consensus statement on catheter and surgical ablation of atrial fibrillation. Heart Rhythm. 2017;14(10):e275-e444. doi:10.1016/j.hrthm.2017.05.012

23. Matsuo S, Yamane T, Date T, Hioki M, Narui R, Ito K, et al. Completion of mitral isthmus ablation using a steerable sheath: prospective randomized comparison with a nonsteerable sheath. J Cardiovasc Electrophysiol. 2011;22:1331-8.

24. Naniwadekar A, Koruth J. Impact of Technique and Technology on Mitral Isthmus Ablation. Curr Treat Options Cardiovasc Med. 2019;21(9):46. Published 2019 Jul 25. doi:10.1007/s11936-019-0752-1

25. Richter B, Gwechenberger M, Filzmoser P, Marx M, Lercher P, Gössinger HD. Is inducibility of atrial fibrillation after radio frequency ablation really a relevant prognostic factor?. Eur Heart J. 2006;27(21):2553-2559. doi:10.1093/eurheartj/ehl307

26. Leong-Sit P, Robinson M, Zado ES, et al. Inducibility of atrial fibrillation and flutter following pulmonary vein ablation. J Cardiovasc Electrophysiol. 2013;24(6):617-623. doi:10.1111/jce.12088

27. Miyazaki S, Shah AJ, Hocini M, Haïssaguerre M, Jaïs P. Recurrent spontaneous clinical perimitral atrial tachycardia in the context of atrial fibrillation ablation. Heart Rhythm. 2015;12(1):104-110. doi:10.1016/j.hrthm.2014.09.055

28. Miyazaki S, Shah AJ, Jadidi AS, et al. Instantaneous electrophysiological changes characterizing achievement of mitral isthmus linear block. J Cardiovasc Electrophysiol. 2011;22(11):1217-1223. doi:10.1111/j.1540-8167.2011.02107.x

29. Maurer T, Metzner A, Ho SY, Wohlmuth P, Reissmann B, Heeger C, et al. Catheter ablation of the superolateral mitral isthmus line: a novel approach to reduce the need for epicardial ablation. Circ Arrhythm Electrophysiol. 2017;10(10). https://doi.org/10.1161/ CIRCEP.117.005191.

30. Ammar S, Luik A, Hessling G, Bruhm A, Reents T, Semmler V, et al. Ablation of perimitral flutter: acute and long-term success of the modified anterior line. Europace. 2015;17(3):447-52. https://doi.org/10. 1093/europace/euu297.

31. Ioannidis P, Zografos T, Vassilopoulos C, et al. The evolution of mapping and ablation techniques in the treatment of atrial tachycardias occurring after atrial fibrillation ablation [published online ahead of print, 2020 May 13]. J Interv Card Electrophysiol. 2020;10.1007/s10840-020-00759-1. doi:10.1007/s10840-020-00759-1

32. Choi Y, Kim S, Baek JY, et al. Acute and long-term outcome of redo catheter ablation for recurrent atrial tachycardia and recurrent atrial fibrillation in patients with prior atrial fibrillation ablation [published online ahead of print, 2020 Jun 16]. J Interv Card Electrophysiol. 2020;10.1007/s10840-02000795-x. doi:10.1007/s10840-020-00795-x

33. Mujović N, Marinković M, Marković N, et al. Persistency of left atrial linear lesions after radiofrequency catheter ablation for atrial fibrillation: Data from an invasive follow-up electrophysiology study. J Cardiovasc Electrophysiol. 2017;28(12):1403-1414. doi:10.1111/jce.13322.

\section{TABLES}

Table 1 . Patient baseline characteristics

\begin{tabular}{llll}
\hline & Total $(\mathrm{n}=72)$ & Clinical Arrhythmia AF $(\mathrm{n}=40)^{*}$ & Clinical Arrhythmia A \\
\hline Age (years) & $62.4 \pm 10.1$ & $61.2 \pm 11.4$ & $63.9 \pm 8.3$ \\
Male gender, n (\%) & $48(66.7 \%)$ & $27(67.5 \%)$ & $21(65.6 \%)$ \\
Arterial hypertension, n $(\%)$ & $44(61.1 \%)$ & $25(62.5 \%)$ & $19(59.4 \%)$
\end{tabular}




\begin{tabular}{llll}
\hline & Total $(\mathrm{n}=72)$ & Clinical Arrhythmia AF $(\mathrm{n}=40)^{*}$ & Clinical Arrhythmia A \\
\hline Diabetes mellitus, $\mathrm{n}(\%)$ & $6(8.3 \%)$ & $3(7.5 \%)$ & $3(9.4 \%)$ \\
Coronary artery disease, $\mathrm{n}(\%)$ & $8(11.1 \%)$ & $2(5 \%)$ & $6(18.8 \%)$ \\
Pacemaker or ICD, n(\%) & $3(4.2 \%)$ & $1(2.5 \%)$ & $3(9.4 \%)$ \\
Left ventricular ejection fraction $<45 \%, \mathrm{n}(\%)$ & $8(11.1 \%)$ & $6(15 \%)$ & $2(6.3 \%)$ \\
Left atrial diameter (mm) & $43.7 \pm 2.7$ & $43.7 \pm 2.8$ & $43.8 \pm 2.6$ \\
Left ventricular ejection fraction (\%) & $52 \pm 5.5$ & $51.5 \pm 4.7$ & $52.7 \pm 6.5$ \\
$\mathrm{CHA}_{2} \mathrm{DS}_{2}$-VASc score & $1.86 \pm 1.28$ & $1.8 \pm 1.29$ & $1.94 \pm 1.29$ \\
Previous AF ablation, n(\%) & & & \\
Total patients, n(\%) & $37(51.4 \%)$ & $9(22.5 \%)$ & $28(87.5 \%)$ \\
Patients with 1 previous procedure, $\mathrm{n}(\%)$ & $33(45.8 \%)$ & $8(20 \%)$ & $3(78.1 \%)$ \\
Patients with 2 previous procedures, n(\%) & $4(5.5 \%)$ & $1(2.5 \%)$ & \\
Type of AF at the time of MI ablation & & & 0 \\
Persistent AF, n(\%) & $21(29.2 \%)$ & $21(52.5 \%)$ & 0 \\
Long-standing persistent AF, n(\%) & $16(22.2 \%)$ & $16(40 \%)$ & 0 \\
Paroxysmal AF, n(\%) & $3(4.2 \%)$ & $3(7.5 \%)$ & \\
\hline
\end{tabular}

*This category includes also the 5 patients with AT occurred during ablation, but had AF as a clinical arrhythmia. AF: Atrial fibrillation, AT: Atrial tachycardia.

Table 2 . Patient characteristics and outcome according to the interventional outcome of MI ablation.

\begin{tabular}{|c|c|c|c|c|c|}
\hline & Total $(\mathrm{n}=72)$ & $\begin{array}{l}\text { Group A } \\
\text { Successful* MI }^{*} \text { ablation without } \\
\text { PMF Recurrence } \\
(\mathrm{n}=62)\end{array}$ & $\begin{array}{l}\text { Group B } \\
\text { Unsuccessful MI } \\
\text { ablation** }(\mathrm{n}=5)\end{array}$ & $\begin{array}{l}\text { Group C PMF } \\
\text { with MI } \\
\text { pseudo-block } \\
(\mathrm{n}=5)\end{array}$ & $\mathrm{P}$ Value \\
\hline \multicolumn{6}{|l|}{$\begin{array}{l}\text { Pre- } \\
\text { procedural } \\
\text { characteris- } \\
\text { tics }\end{array}$} \\
\hline Age (years) & $62.4 \pm 10.1$ & $62.5 \pm 10.2$ & $66 \pm 12.7$ & $57 \pm 5.8$ & 0.3652 \\
\hline $\begin{array}{l}\text { Male gender, } \mathrm{n} \\
(\%)\end{array}$ & $45(62.5 \%)$ & $37(59.7 \%)$ & $5(100 \%)$ & $3(60 \%)$ & 0.1995 \\
\hline $\begin{array}{l}\text { Left atrial } \\
\text { diameter }(\mathrm{mm})\end{array}$ & $43.7 \pm 2.7$ & $43.3 \pm 2.4$ & $48 \pm 2^{+}$ & $44.2 \pm 2.9$ & 0.0004 \\
\hline $\begin{array}{l}\text { Left } \\
\text { ventricular } \\
\text { ejection } \\
\text { fraction }(\%)\end{array}$ & $52 \pm 5.5$ & $52.4 \pm 4.6$ & $47 \pm 13$ & $52 \pm 4.5$ & 0.1107 \\
\hline $\begin{array}{l}\mathrm{CHA}_{2} \mathrm{DS}_{2^{-}} \\
\text {VASc } \\
\text { score }\end{array}$ & $1.86 \pm 1.28$ & $1.87 \pm 1.27$ & $2.4 \pm 1.52$ & $1.2 \pm 1.09$ & 0.3327 \\
\hline $\begin{array}{l}\text { Previous AF } \\
\text { ablation, } \mathrm{n}(\%) \\
\text { Clinical } \\
\text { arrhythmia at } \\
\text { the time of } \\
\text { procedure }\end{array}$ & $37(51.4 \%)$ & $29(46.8 \%)$ & $4(80 \%)$ & $4(80 \%)$ & 0.1492 \\
\hline $\mathrm{AF}, \mathrm{n}(\%)$ & $40(55.6 \%)$ & $38(61.3 \%)$ & $1(20 \%)$ & $1(20 \%)$ & 0.0512 \\
\hline
\end{tabular}




\begin{tabular}{|c|c|c|c|c|c|}
\hline & Total $(\mathrm{n}=72)$ & $\begin{array}{l}\text { Group A } \\
\text { Successful* MI } \\
\text { ablation without } \\
\text { PMF Recurrence } \\
(\mathrm{n}=62)\end{array}$ & 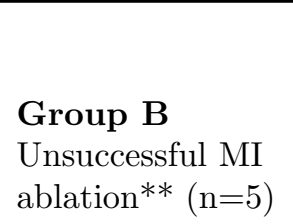 & $\begin{array}{l}\text { Group C PMF } \\
\text { with MI } \\
\text { pseudo-block } \\
(\mathrm{n}=5)\end{array}$ & $\mathrm{P}$ Value \\
\hline $\begin{array}{l}\mathrm{AT}, \mathrm{n}(\%) \\
\text { Procedural } \\
\text { characteris- } \\
\text { tics }\end{array}$ & $32(44.4 \%)$ & $24(38.7 \%)$ & $4(80 \%)$ & $4(80 \%)$ & \\
\hline $\begin{array}{l}\text { Epicardial } \\
\text { approach }, \\
\mathrm{n}(\%) \\
\text { MI ablation } \\
\text { time (min) }\end{array}$ & $33(45.8 \%)$ & $28(45.2 \%)$ & $3(60 \%)$ & $2(40 \%)$ & 0.7851 \\
\hline Total & $10.73 \pm 6.16$ & $10.73 \pm 6.14$ & $18.7 \pm 9.51^{++}$ & $10.16 \pm 2.93$ & 0.0262 \\
\hline Endocardial & $10.12 \pm 5.87$ & $10.27 \pm 5.86$ & $17.66 \pm 8.54^{++}$ & $9.96 \pm 3.14$ & 0.0313 \\
\hline $\begin{array}{l}\text { Epicardial } \\
\text { MI line } \\
\text { dimensions }\end{array}$ & $1 \pm 0.54$ & $0.96 \pm 0.49$ & $1.73 \pm 0.46^{++++}$ & $0.5 \pm 0.42$ & 0.0005 \\
\hline Length (mm) & $32.0 \pm 4.3$ & $31.7 \pm 4.2$ & $36.2 \pm 1.8$ & $32.2 \pm 5.0$ & 0.0735 \\
\hline $\begin{array}{l}\text { Width }(\mathrm{mm}) \\
\text { Post- } \\
\text { procedural } \\
\text { characteris- } \\
\text { tics and } \\
\text { outcome }\end{array}$ & $13.8 \pm 4$ & $13.7 \pm 3.6$ & $18.6 \pm 6.6^{\S}$ & $11.2 \pm 3.6$ & 0.0088 \\
\hline $\begin{array}{l}\text { Follow-up } \\
\text { period } \\
\text { (months) }\end{array}$ & $22.2 \pm 8.1$ & $21.7 \pm 8.4$ & $27.2 \pm 6.1$ & $24.6 \pm 4.9$ & 0.2845 \\
\hline $\begin{array}{l}\mathrm{AT} / \mathrm{AF} \\
\text { recurrence, } \\
\mathrm{n}(\%)\end{array}$ & $16(22.2 \%)$ & $13(21 \%)$ & $2(40 \%)$ & $1(20 \%)$ & 0.6111 \\
\hline $\begin{array}{l}\mathrm{AT} \text { or } \\
\text { mainly }{ }^{\S} \mathrm{AT} \\
\text { recurrence, } \\
\mathrm{n}(\%)\end{array}$ & $2(2.8 \%)$ & $2(3.2 \%)$ & 0 & 0 & 0.7682 \\
\hline $\begin{array}{l}\mathrm{AF} \text { or } \\
\text { mainly }{ }^{\S} \mathrm{AF} \\
\text { recurrence, } \\
\mathrm{n}(\%)\end{array}$ & $14(19.4 \%)$ & $11(17.8 \%)$ & $2(40 \%)$ & $1(20 \%)$ & \\
\hline $\begin{array}{l}\text { Post- } \\
\text { procedural } \\
\text { antiarrhythmic } \\
\text { medication } \\
\mathrm{n}(\%)\end{array}$ & $9(12.5 \%)$ & $6(9.7 \%)$ & $2(40 \%)$ & $1(20 \%)$ & 0.1246 \\
\hline $\begin{array}{l}\text { Amiodarone, } \\
\mathrm{n}(\%)\end{array}$ & $5(6.9 \%)$ & $3(4.8 \%)$ & $2(40 \%)$ & 0 & 0.2317 \\
\hline Other, $\mathrm{n}(\%)$ & $4(5.6 \%)$ & $3(4.8 \%)$ & 0 & $1(20 \%)$ & \\
\hline
\end{tabular}

*After the 1st MI ablation attempt. **Unsuccessful MI ablation after the 1st procedure $(\mathrm{n}=4)$ or PMF 
occurrence due to overt MI conduction recurrence $(n=1)$. +Group B vs. Group A, $\mathrm{p}=0.0002$; Group B vs. Group C, p=0.0396, ++Group B vs. Group A, p=0.0209, ++Group B vs. Group A, p=0.0246, ++++ Group B vs. Group A, p= 0.003; Group B vs. Group C, p=0.0004, ${ }^{\S}$ Group B vs. Group A, p=0.0207; Group B vs. Group C, $p=0.0090 .{ }^{\S}$ More than $50 \%$ of the documented arrhythmia burden. \#Systemic AAD therapy, over a period of more than 3 months within the follow-up period (excluding the first 3 months of the blanking period). AF: Atrial fibrillation, AT: Atrial tachycardia, MI: Mitral isthmus, PMF: Perimitral atrial flutter.
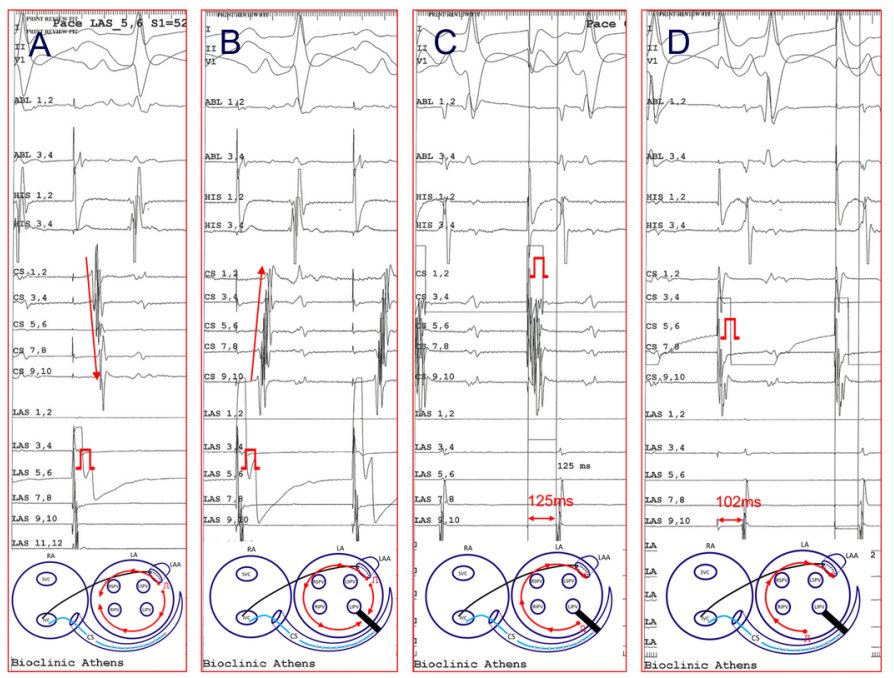

\section{FIGURES}

Figure 1. The pacing maneuvers performed in all patients to confirm MI bidirectional block. Pacing superiorly to MI line before (A) and after (B) ablation to prove conduction block in the clockwise direction. Differential pacing from CS to prove conduction block in counterclockwise direction (C,D).

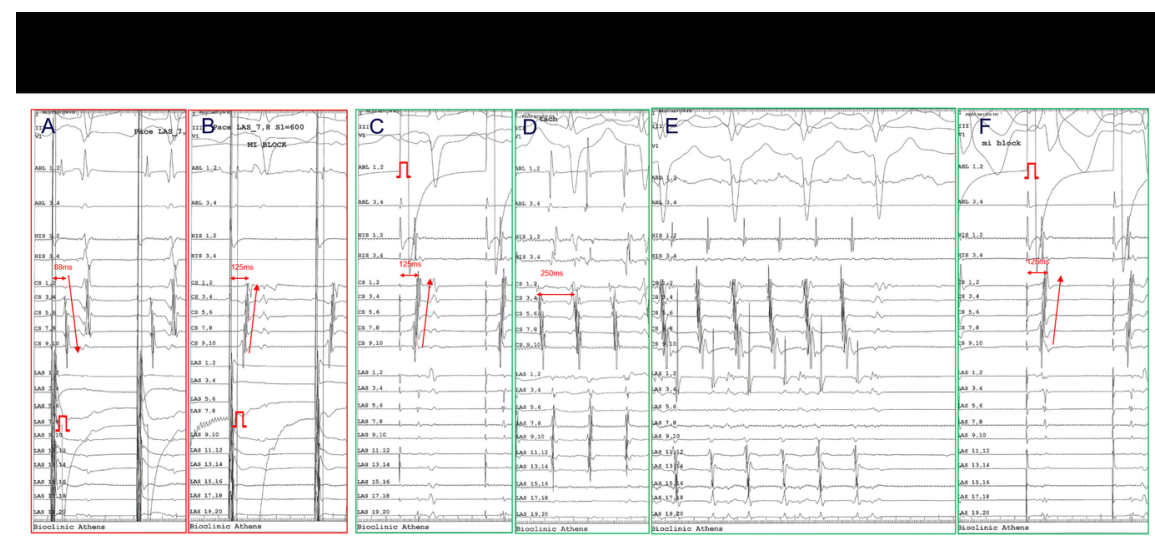


Figure 2. Pacing maneuvers indicative of MI block and PMF. A: Pacing from LAA with MI conduction before MI ablation in the $1^{\text {st }}$ procedure. B. Pacing from LAA with complete reversal in CS sequence after MI ablation in the $1^{\text {st }}$ procedure. C: Pacing superiorly of the MI line at the beginning of the $2^{\text {nd }}$ procedure before any ablation. D: Clockwise PMF with $\mathrm{CL}=250 \mathrm{~ms}$. E: Termination of the arrhythmia by ablating the gap in MI (Video 3). F: Reconfirmation of MI block.
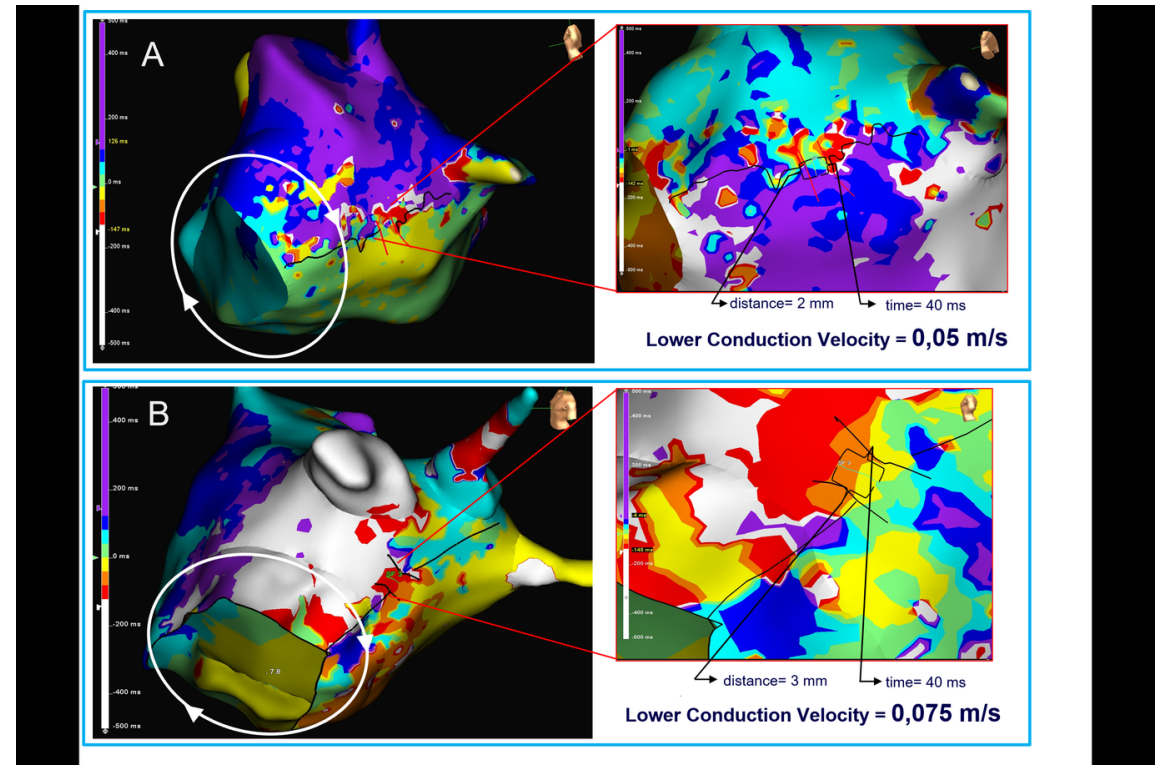

Figure 3 . Isochronal maps in two cases (A, B) of clockwise PMF circuits and MI pseudo-block showing the impulse breakthrough from a narrow channel in the MI with very low conduction velocity (Videos 1 and 4 , respectively).
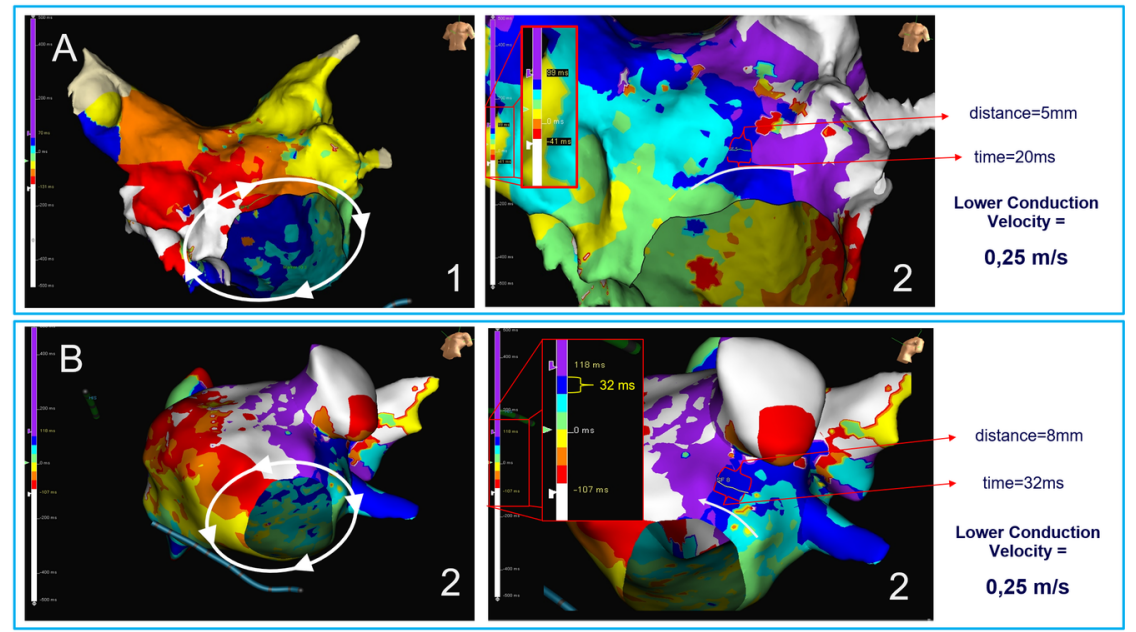

Figure 4. Isochronal maps of clockwise (A1) and counterclockwise (B1) PMF with the corresponding measurement of the lower CV in each circuit (A2, B2) (Video 5,6). 


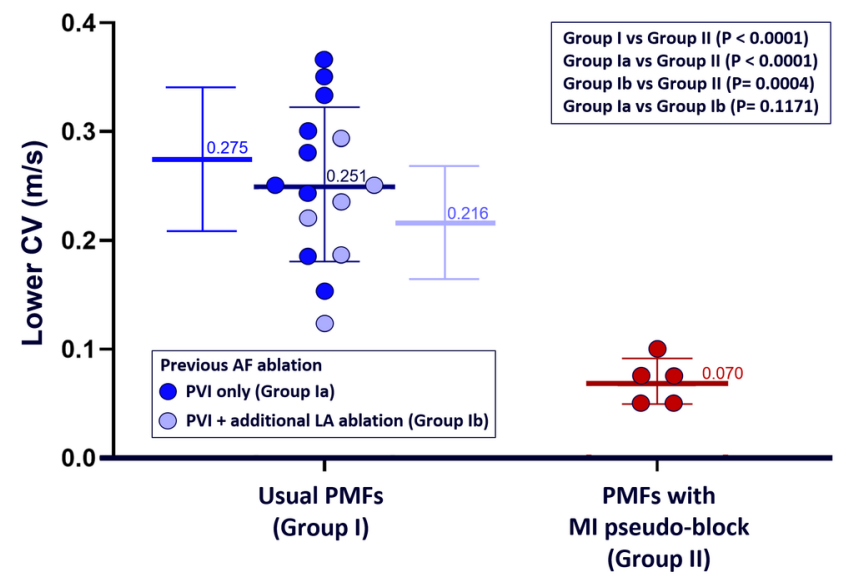

Figure 5. Comparison of the lower CVs in usual PMF circuits and in PMF circuits after confirmed MI block.
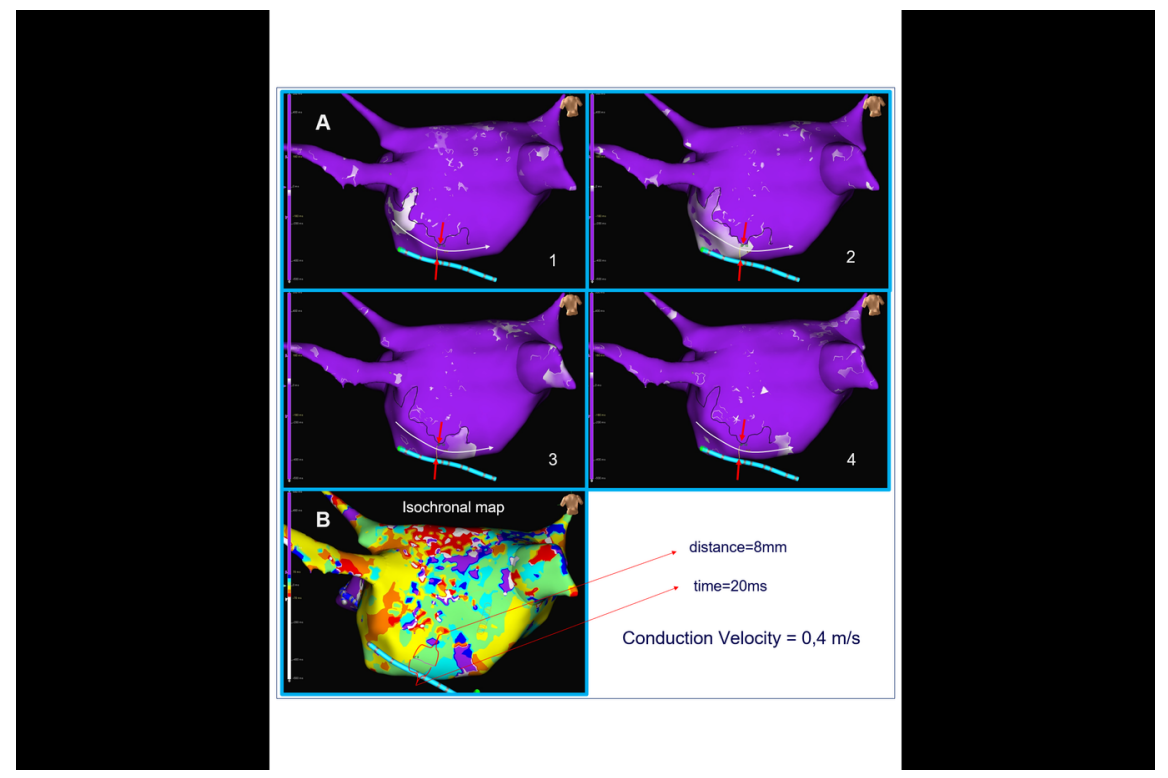

Figure 6. Propagation map of a clockwise PMF with a narrow conduction channel (red arrows) in the lower mitral annulus (video 7 ). The CV in this area was manually measured $0.4 \mathrm{~m} / \mathrm{s}$. 

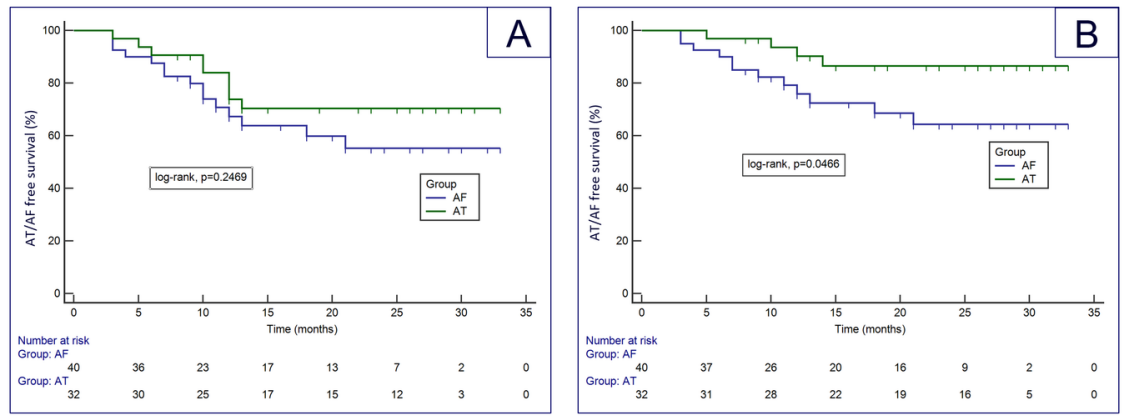

Figure 7. Kaplan-Meier curve of AT/AF free survival in patients with AT or AF after the index MI ablation (A) and the subsequent redo procedures (B).
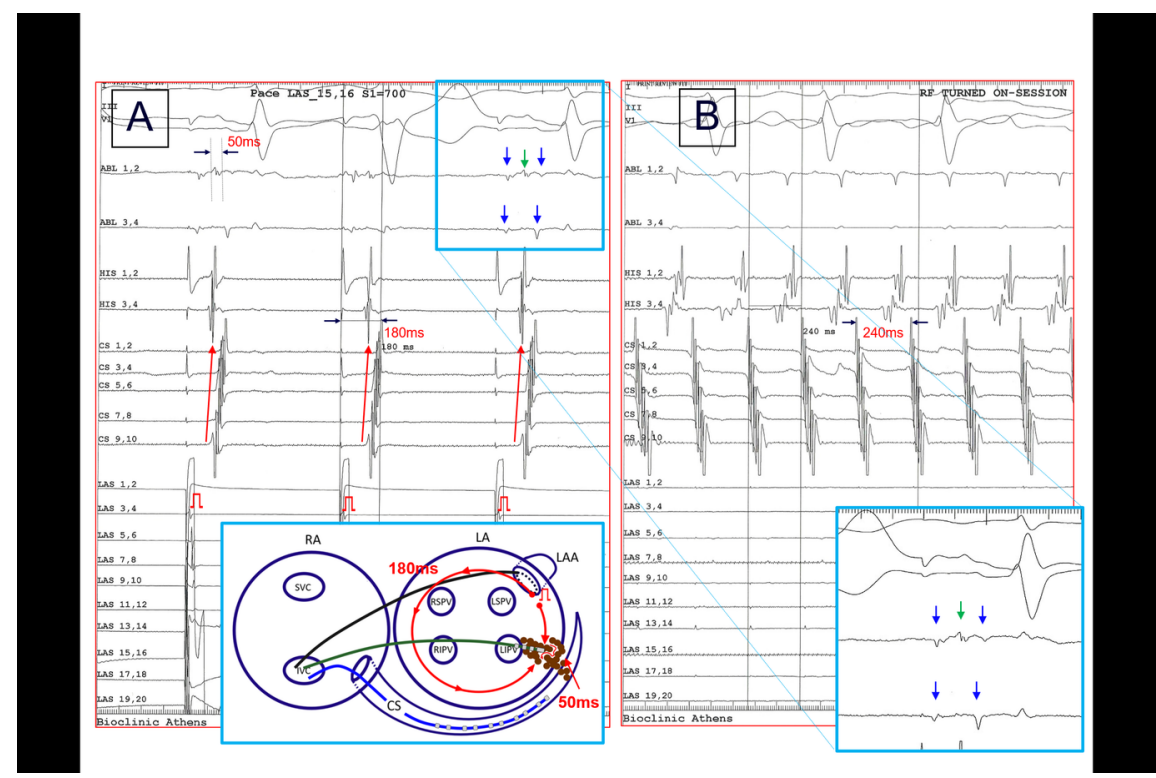

Figure 8. A: Pacing from the LAA showing the reversal of the activation sequence in the CS. The time of the counterclockwise rotation from the spike to the distal CS was $180 \mathrm{~ms}$. The ablation catheter is located in the MI line and records double far-field potentials indicative of conduction block (blue arrows) and in between a high frequency near-field electrogram (green arrow) with total duration 50ms. The latter is possibly indicative of residual conduction through the MI ablation area, it does not have enough time to cross the MI and eventually fades colliding with the counterclockwise wavefront.B: Clockwise PMF with CL $240 \mathrm{~ms}$, that was diagnosed with high density activation mapping using the 3D mapping system. 